\title{
Combining proprioception and touch to compute spatial information
}

\author{
Elisa Canzoneri • Elisa Raffaella Ferrè • \\ Patrick Haggard
}

Received: 21 August 2013 / Accepted: 13 January 2014 / Published online: 28 January 2014

(C) Springer-Verlag Berlin Heidelberg 2014

\begin{abstract}
Localising a tactile stimulus in egocentric space involves integrating information from skin receptors with proprioceptive inputs about body posture. We investigated whether body posture automatically influences tactile spatial judgements, even when it is irrelevant to the task. In Experiment 1, participants received two successive tactile stimuli on the forearm and were asked to indicate whether the first or second touch of the pair was closer to an anatomical body landmark, either the wrist or the elbow. The task was administered in three experimental conditions involving different body postures: canonical body posture with extended forearm and hand pointing distally; a noncanonical body posture with forearm and hand pointing vertically up at $90^{\circ}$ and a 'reversed' body posture with the elbow fully flexed at $180^{\circ}$, so that the hand pointed proximally. Thus, our task required localising touch on the skin and then relating skin locations to anatomical body landmarks. Critically, both functions are independent of the posture of the body in space. We nevertheless found reliable effects of body posture: judgement errors increased when the canonical forearm posture was rotated through $180^{\circ}$. These results were further confirmed in Experiment 2 , in which stimuli were delivered to the finger. However,
\end{abstract}

E. Canzoneri · E. R. Ferrè · P. Haggard

Institute of Cognitive Neuroscience, University College London, Alexandra House, Queen Square, London WC1N 3AR, UK

E. Canzoneri

Centro Studi e Ricerche in Neuroscienze Cognitive, University of Bologna, Viale Europa 980, Cesena, Italy

Present Address:

E. Canzoneri $(\square)$

Ecole Polytechnique Fédérale de Lausanne, Route Cantonale, 1015 Lausanne, Switzerland

e-mail: elisa.canzoneri3@unibo.it additionally reversing the canonical posture of the finger, as well as that of the forearm, so that the finger was restored to its canonical orientation in egocentric space, restored performance to normal levels. Our results confirm an automatic process of localising the body in external space underlying the process of tactile perception. This process appears to involve a combination of proprioceptive and tactile information.

Keywords Tactile localisation · Body space . Body model $\cdot$ Proprioception

\section{Introduction}

When a stimulus enters in contact with our skin, we immediately know where we have been touched. Thus, a cardinal aspect of somatosensory representation is the ability to localise touch. Somatosensory receptors in the skin project to the well-known sensory homunculus of the primary somatosensory cortex, which forms a characteristically distorted map of the contralateral body surface (S1, Penfield and Rasmussen 1950). This map is often thought sufficient in itself for perception of tactile location. However, localising tactile inputs on the skin, or within the somatotopic map, is not sufficient for a complete process of localisation. First, the relation between the skin and the underlying body can vary slightly as the skin moves over the underlying musculoskeletal structure. Second, and more prominently, body parts such as the limbs are in constant movement relative to the body core so that the position of a tactile stimulus in egocentric external space may vary.

Therefore, localising stimuli on the skin requires a multistage process, involving a number of different body representations (Longo et al. 2010). First, the stimulus-evoked 
activity is represented within the somatotopic map or 'superficial schema' (Head and Holmes 1911). This way, as soon as a stimulus touches our skin, we know which part of the skin has been touched. Second, the skin region is related to the underlying body structure, e.g. the hand, to provide a somatotopic location. Third, the somatotopic location is mapped onto a location in egocentric external space where the underlying body part is currently located (Röder et al. 2004; Yamamoto and Kitazawa 2001a). Localising a tactile stimulus in external space therefore requires integrating information from somatosensory receptors with proprioceptive inputs about body posture (Longo et al. 2010). In contrast, localising a stimulus with respect to an underlying musculoskeletal landmark requires only information from somatosensory receptors and registration of the skin with the underlying body structures.

The online information about body part posture has been called the 'postural schema' (Head and Holmes 1911; Longo et al. 2010). This integration between somatosensory and proprioceptive information allows tactile stimulus to be remapped into external space (Azanon et al. 2010a, b). Critically, a strong dissociation occurs between somatotopic and external frames of reference. The former are relatively stable, while the latter are constantly updated when body parts moves.

Recent psychophysical studies suggested that tactile remapping from skin space to external space is performed automatically, even for purely tactile processing, such as steps 1 and 2 of the model above (Longo et al. 2010), when external spatial coordinates are not needed. Put in another way, spatial reference frames appear to dominate over cutaneous and somatotopic ones. Several experimental studies have investigated the changing of reference frames during tactile encoding by comparing the processing of identical tactile stimuli delivered in different body postures. In particular, crossing the arms over the midline has been extensively used to study tactile spatial encoding. Yamamoto and Kitazawa (2001a) used a temporal order judgement task to investigate how body posture interferes with tactile temporal order. Their participants easily reported the temporal order of tactile stimulation delivered to each hand when the hands were in the normal, uncrossed posture. However, crossing the hands over the midline changed the temporal order judgement, and participants frequently inverted the temporal order of the two stimuli. Critically, these reversal errors occurred only at short temporal intervals between the two stimuli (300 ms), but not at longer intervals (i.e. more then $1,000 \mathrm{~ms}$ ). This suggests that the probability of reversal is strictly dependent on the interval between the two touches.

The crossed hands deficit may reflect conflict between two concurrently active reference frames: one somatotopic and one external. Accordingly, Azanon and Soto-Faraco (2008a), using a crossmodal cueing paradigm, demonstrated that the very earliest information about tactile location involves a somatotopic frame of reference. Later, the external frame of reference prevails, as measured by a progressive impact of the crossed hands posture as the interval between a tactile and visual stimulus is increased. The process of remapping spatial coordinates was completed in an interval ranging from $180 \mathrm{~ms}$ to $360 \mathrm{~ms}$ after stimulus onset (Azanon and Soto-Faraco 2008a).

Most of the existing studies have focused on left/right division of space. Touches are delivered to both hands, in crossed and uncrossed postures. However, crossing the hands over the midline of the body not only involves a postural component, but also other, less specific effects, such as the left/right segmentation of space (Bisiach et al. 1986; Husain 2008), and the possibility of confusion between verbal response labels 'left' and 'right'. Indeed, a simple confusion between response labels could result in a crossed hand deficits in a temporal order judgement task, without implying any difficulty in spatial perception of touch.

Here, we aimed to investigate the effects of body posture on tactile perception, within a single limb and a single hemisphere. We designed a version of the tactile temporal order judgement tasks in which external spatial frames of reference were entirely irrelevant. First, both stimuli were delivered to a single side of the body. This way, our experimental task can be conceived as monohemispheric, allowing us to investigate the effect of body posture on tactile perception within a single hemisphere. Second, the limbs never crossed the midline. Third, the response categories made no reference to external spatial labels such as 'left' and 'right', but referred only to whether the skin overlying a designated body landmark was stimulated first or second in the pair. Critically, our task made no reference to spatial constructs other than body parts. If limb posture influenced tactile judgement under these conditions, this would provide powerful evidence of an automatic remapping of skin stimuli into egocentric external space by changes in body posture. Step 3 of the tactile localisation process model, as presented by Longo et al. (2010), involves remapping of a tactile stimulus from a somatotopic frame of reference to a location in egocentric external space. This remapping would be automatic and would dominate tactile perception, even when irrelevant to the task and detrimental to performance. In Experiment 1, we investigated whether two successive tactile stimuli administered on the forearm were coded in a different way according to the posture of the forearm in space. In Experiment 2, we investigated the combined effects of arm and finger posture on temporal order judgements for stimuli on the finger. Importantly, Experiment 2 allows us to rule out explanations based on postural discomfort or awkwardness. 


\section{Experiment 1}

Participants

Twelve healthy participants (seven males, mean age \pm standard deviation (SD): $25.4 \pm 4.8$ years) took part for payment in the study and on the basis of written informed consent. Subjects with a history of sensory or neurological disorders were excluded. All participants were right handed as assessed using the Edinburgh handedness inventory. The experimental protocol was approved by the research ethics committee of University College London. The study was designed according to ethical standards of the Declaration of Helsinki.

\section{Experimental procedure}

Blindfolded participants sat with their left arm extended on a table placed into the left of the midline. The table height was adjusted with the participant's shoulder. Tactile stimulation consisted of a stimulus with supra-threshold intensity delivered by two solenoid tappers (RS 330-5213 solenoids, uk.rs-online.com) applied on the dorsal surface of the left forearm along the Distal-Proximal axis (Fig. 1). The distance between the two solenoids tappers was clearly above each participant's spatial acuity threshold, based on twopoint discrimination thresholds (2pdt). To establish discrimination threshold, the experimenter touched the skin location with a definitively supra-threshold von Frey nylon filament mounted on a calliper. Either double (70\%) or single touches $(30 \%)$ were administered at random. Only double touches were used to compute the staircase. The calliper was applied on the forearm with pressure sufficient to first blanch the skin. The starting separation was $40 \mathrm{~mm}$, above the 2pdt for this skin region (Weinstein 1968). The participant was instructed to say 'one', when one point was felt, or 'two', when two points were felt. The separation was reduced progressively by $50 \%$ after each set of three successive correct responses. When participants made three consecutive errors when testing one distance, the separation was subsequently increased to midpoint of the current (erroneous) trial and the immediately preceding (correct) trial. We assumed that the shortest separation at which participant perceived two touches could be considered an estimate for 2 pdt. We then confirmed this estimate by delivering five double touches at this separation, randomly intermixed with five single touches. If participants scored between $7 / 10$ and $9 / 10$ correct, this threshold estimate was accepted for experimental testing. Otherwise, the procedure was repeated.

Each participant performed the task in three different experimental conditions (Fig. 1). In one condition, the arm was placed on the table palm down in the canonical

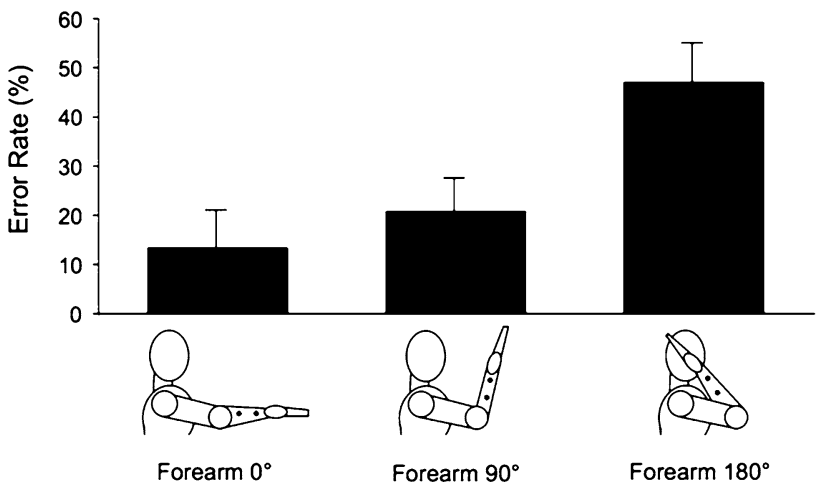

Fig. 1 Experiment 1: experimental conditions and results. Mean error rate $(\%)$ in function of the three different postural conditions: Forearm $0^{\circ}$ Posture, Forearm $90^{\circ}$ Posture and Forearm $180^{\circ}$ Posture. Error bars indicate SEM. Note a significant increase in the error rate for the Forearm $180^{\circ}$ Posture

posture, with hand and finger pointing distally (Forearm $0^{\circ}$ Posture). In another condition, the arm was placed on the table, but the forearm and the hand tilted to point up at $90^{\circ}$ (Forearm $90^{\circ}$ Posture). In the last condition, the arm was placed on the table, but the elbow was fully flexed so that the forearm was reversed, with the hand pointed proximally (Forearm $180^{\circ}$ Posture). For each participant, 2pdts were measured separately for each arm posture. The intersolenoid distance used during the experimental task was set to be 1.5 , the corresponding $2 \mathrm{pdt}$. The $2 \mathrm{pdt}$ was assessed again at the end of the experiment for each arm position, in order to investigate any possible change in tactile acuity. Moreover, during the task, participants were asked to verbally confirm that they were able to clearly perceive two separate taps for each trial.

Two different interstimulus intervals (ISI) were used between a pair of taps: 150 and $550 \mathrm{~ms}$. These intervals were chosen on the basis of previous tactile motion studies conducted on the skin surface of the forearm, to clearly evoke an intermediate motion perception $(150 \mathrm{~ms}$, Flach and Haggard 2006) or instead to evoke the sensations of two separate taps, without intermediate motion $(550 \mathrm{~ms}$; see Flach and Haggard 2006 for data on the forearm surface). In half of the trials, at random, the proximal solenoid, located close to the elbow, was activated first. The distal solenoid, located close to the wrist, was activated first on the remaining trials. This resulted in two possible directions of tactile stimulation (Proximal-Distal and Distal-Proximal). Participants were asked to discriminate which stimulus was closer to the wrist (or to the elbow), saying 'First' or 'Second'. The body cue (wrist, elbow) was counterbalanced between participants, with half participants performing the task using the wrist as body cue and the remaining participants using the elbow. Participants were reminded of the relevant cue at the beginning of the block. Participants were verbally 
Table 1 Experiment 1: errors

\begin{tabular}{llllll}
\hline & \multicolumn{2}{l}{ Distal to proximal } & & \multicolumn{2}{l}{ Proximal to distal } \\
\cline { 2 - 3 } \cline { 5 - 6 } & 150 & 550 & & 150 & 550 \\
\hline $\begin{array}{l}\text { Forearm } 0^{\circ} \\
\text { Posture }\end{array}$ & $18 \pm 6.16$ & $12 \pm 3.18$ & & $18.50 \pm 5$ & $14 \pm 6.23$ \\
$\begin{array}{l}\text { Forearm } 90^{\circ} \\
\text { Posture }\end{array}$ & $15 \pm 5.11$ & $16.5 \pm 5.87$ & & $40 \pm 5.82$ & $19.5 \pm 5.84$ \\
$\begin{array}{l}\text { Forearm } 180^{\circ} \\
\quad\end{array}$ & $49 \pm 9.18$ & $42.5 \pm 7.24$ & $52 \pm 8.63$ & $44.5 \pm 10.26$ \\
& & & & \\
\hline
\end{tabular}

Mean (SEM) error rate (\%) in each experimental condition

instructed at the beginning of each block about the arm posture to maintain throughout the block, and the order of postures was randomly chosen between participants. The experimenter checked that the arm posture was correct. In particular, the distance between participants' arm and their head was maintained constant in the different conditions. Participants performed two blocks of 40 trials each for each arm posture, resulting in a total of 120 trials. The two different ISIs (150-550 ms) and the direction of tactile stimulation (Proximal-Distal, Distal-Proximal) were randomly chosen and controlled by a computer. White noise was presented through headphones during the trial to mask any auditory cues from the tactile stimulation.

\section{Results}

For each participant, we estimated error rates separately for each arm posture. The raw data in each condition are shown in Table 1. The number of data points at each ISI and the number of ISIs were too small to reliably fit psychometric functions.

These probabilities were entered in a repeated-measure ANOVA with Arm Posture (Forearm $0^{\circ}$, Forearm $90^{\circ}$, Forearm $180^{\circ}$ ), Direction (Proximal-Distal, Distal-Proximal) and ISI $(150,550 \mathrm{~ms})$ as within subject factors. Arm Posture affected participants' responses, as shown by the significant main effect of Arm Posture $[F(2,18)=9.12, p=0.001]$. Participants made more errors in the Forearm $180^{\circ}$ Posture (error rate: $47 \%$ ) than in either the Forearm $0^{\circ}$ Posture (error rate: $16 \%, p<0.01$ ) or the Forearm $90^{\circ}$ Posture (error rate: $23 \%$, $p<0.01$, Newman-Keuls corrected). Indeed, performance in the $180^{\circ}$ Posture did not exceed chance levels $(p=0.73)$. We also found an effect of Direction $[F(1,9)=9.09$, $p=0.01]$, with more errors in the Distal-Proximal direction (error rate: $31 \%$ ) as compared to the Proximal-Distal one (error rate: $26 \%, p<0.05$ ). A significant Posture $\times$ Direction interaction $[F(2,18)=5.14, p=0.01]$ showed that the higher probability of error in the Distal-Proximal direction was specific for the Forearm $90^{\circ}$ Posture (Distal-Proximal direction error rate: $30 \%$; Proximal-Distal direction error rate: $16 \%, p<0.001$ ), but not for the other arm postures. Finally, as expected, participants made more errors at shorter than longer ISIs (150 ms error rate: $32 \% ; 550 \mathrm{~ms}$ error rate: $25 \%$ ), producing a significant main effect of ISI $[F(1,9)=11.02, p=0.008]$. Critically, the ISI did not interact with Arm Posture $[F(2,18)=0.45, p=0.65]$, suggesting that the higher probability of error in the Forearm $180^{\circ}$ Posture was not dependent on the ISI, but mainly on the posture adopted during the task.

\section{Discussion}

Results from this experiment showed that temporal order judgement gradually deteriorated as the arm posture shifted away from its canonical posture, towards a rotated, and then a reversed posture. In the fully reversed posture (Forearm $180^{\circ}$ Posture), information about tactile temporal order was effectively lost, and performance was at chance level. Interestingly, the postural modulation of error rates was independent of ISI, despite a main effect of ISI in line with previous results. Caution is always required in interpreting null results, particularly from small experiments. However, the current data suggest that postural effects are not simply due to tactile motion perception or to task difficulty.

Importantly, our task made no reference to location of the body parts in external space or to external spatial response categories. Our task required only that participants locate a skin stimulus with respect to the underlying musculoskeletal landmarks. This relation is essentially independent of body posture. Nevertheless, we found a profound impairment when the forearm posture was rotated by $180^{\circ}$. In this condition, the body landmarks occupied transposed locations, with the wrist being closer to the body than the elbow. In contrast, rotating the elbow through $90^{\circ}$ had only a minimal and nonsignificant effect. This pattern of results suggests that the deficit in the $180^{\circ}$ condition may arise because the body landmarks were identified partly on the basis of their respective locations in egocentric external space. Swapping the external spatial locations of the body landmarks led to difficulty in perceiving the temporal order of stimulation on the skin close to them.

In summary, results from this experiment demonstrated that proprioceptive information about the locations of body parts in space is necessary in order to compute the location of a tactile stimulus on the body.

\section{Experiment 2}

\section{Participants}

Eight right-handed healthy participants (four males, mean age \pm SD: $24.2 \pm 3.89$ years) took part in Experiment 2 . 


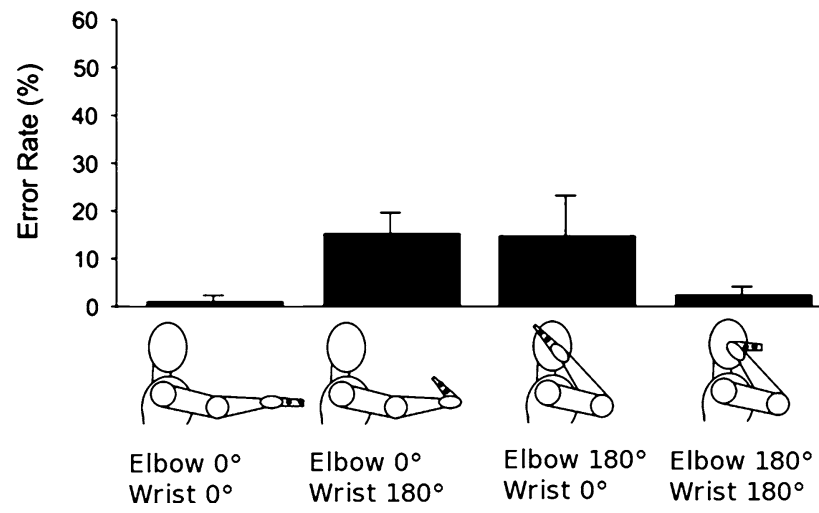

Fig. 2 Experiment 2: experimental conditions and results. Mean error rate $(\%)$ in function of the different postural conditions: Elbow $0^{\circ}$ Wrist $0^{\circ}$, Elbow $0^{\circ}$ Wrist $180^{\circ}$, Elbow $180^{\circ}$ Wrist $0^{\circ}$ and Elbow $180^{\circ}$ Wrist $180^{\circ}$ Posture. Error bars indicate SEM

\section{Experimental procedure}

Two solenoids were placed on the dorsal surface of the left index finger. Given the high tactile acuity on the finger (Weinstein 1968), the distance between the two solenoids was manually set as equal for every participant on the basis of a piloting study: one solenoid was placed $1 \mathrm{~cm}$ proximally from the nail and the other solenoid on the dorsal surface of middle phalanx. This configuration resulted in a intersolenoid distance of approximately $10 \mathrm{~mm}$. All participants clearly perceived two separate taps for each trial.

The postures tested were based on a factorial combination of reversing the posture of the forearm by rotating elbow by $180^{\circ}$ and reversing the direction of the hand by rotating the wrist by $180^{\circ}$. The same procedure administered during Experiment 1 was therefore adapted to four different experimental conditions (Fig. 2). Note that when only the elbow is rotated, or only the wrist is rotated, the index finger points towards the body, causing a conflict between the current posture of the limb in space and the 'normal' canonical spatial frame of reference for the finger. In contrast, when both the elbow and the wrist are rotated, the index finger points in its normal, canonical frame of reference, away from the body. There is thus no conflict between current posture and the external spatial frame of reference, despite the altered internal kinematic configuration of the limb.
Participants were asked to respond 'First' or 'Second' to indicate which stimulus was closer to the index fingertip (or to the index finger knuckle). The order of the body landmark relative to which they judged tactile locations was counterbalanced across participants. Participants performed two blocks (40 trials each) for every experimental condition, resulting in a total of 160 trials. In other aspects, the design resembled Experiment 1.

\section{Results}

For each participant, we calculated the error rates separately for each combination of Elbow angle, Wrist angle, ISI and Direction of tactile stimulus sequence. We averaged over the two directions of tactile stimulus sequence (knuckle first then finger tip, finger tip first then knuckle), because the design of Experiment 2 ensured that this factor was completely orthogonal to the spatial factors of joint angle and posture of the finger in external space. Direction of stimulus sequence was not therefore a factor of interest about which we made specific predictions. The raw data are presented in Table 2. Error rates were analysed using a three-way repeated-measures ANOVA, with factors of Elbow angle, Wrist angle and ISI. We found a predicted trend of ISI $[F(1,7)=4.172, p=0.080]$. There was no significant main effect of Elbow angle $[F(1,7)=0.052$, $p>0.05]$ or of Wrist angle $[F(1,7)=0.122, p>0.05]$. However, there was a significant interaction between Elbow angle and Wrist angle $[F(1,7)=7.247, p=0.031]$. This interaction arose because error rates in the Elbow $180^{\circ}$ and Wrist $180^{\circ}$ condition, when both joints were reversed, restoring the finger to its normal posture in egocentric space, were significantly reduced relative to the Elbow 0 Wrist 180 condition, where only one of the joints was rotated ( $p<0.01$, Bonferroni corrected), and they showed a statistical trend towards reduction as compared to the Elbow 180 Wrist 0 condition $(p=0.07)$ so as to place the finger into a reversed posture in egocentric space. Other interactions were not significant (all $p_{\mathrm{s}}>0.197$ ).

\section{Discussion}

Performance in Experiment 2 was generally better than Experiment 1, perhaps reflecting the greater somatosensory
Table 2 Experiment 2: errors

Mean (SEM) error rate (\%) in each experimental condition

\begin{tabular}{lccccr}
\hline & \multicolumn{2}{c}{ Distal to proximal } & & \multicolumn{2}{c}{ Proximal to distal } \\
\cline { 2 - 3 } \cline { 5 - 6 } & \multicolumn{1}{c}{150} & & 150 & \multicolumn{1}{c}{550} \\
\hline Elbow $0^{\circ}$ Wrist $0^{\circ}$ Posture & $1.88 \pm 1.32$ & $0 \pm 0$ & & $0.63 \pm 0.63$ & $0.63 \pm 0.63$ \\
Elbow $0^{\circ}$ Wrist $180^{\circ}$ Posture & $21.88 \pm 5.17$ & $12.50 \pm 5.18$ & & $14.38 \pm 5.04$ & $14.38 \pm 5.04$ \\
Elbow $180^{\circ}$ Wrist $0^{\circ}$ Posture & $25 \pm 7.26$ & $11.25 \pm 8.49$ & & $13.13 \pm 4.99$ & $13.13 \pm 7.79$ \\
Elbow $180^{\circ}$ Wrist $180^{\circ}$ Posture & $2.50 \pm 1.34$ & $0 \pm 0$ & & $2.50 \pm 0.94$ & $2.50 \pm 1.89$ \\
\hline
\end{tabular}


innervation of the finger, compared to the forearm. Otherwise, results from Experiment 2 confirmed and extended those of Experiment 1. First, we showed that reversing the hand posture by rotating either the elbow or the wrist impaired tactile temporal order judgement. However, the two-way ANOVA interaction between Elbow and Wrist angle showed that a double rotation of both elbow and wrist, which restored the canonical spatial locations of the body landmarks referenced in the temporal order judgement, also restored performance levels to those found for normal posture. Performance levels were generally good, and ceiling effects are a possibility. However, both the impairment due to hand reversal and the remission due to additional wrist reversal involve large, double-digit changes in error rate that are unlikely to be restricted by ceiling effects. Moreover, the critical two-way interaction between Elbow and Wrist angle was, if anything, stronger for the more difficult $150 \mathrm{~ms}$ ISI condition than for the easier $550 \mathrm{~ms}$ ISI condition. That is, the crucial role of stimulus location in external space was strongest farther from ceiling. Thus, any ceiling effect counts against our hypothesis and would lead us to underestimate rather than overestimate the importance of external spatial position in temporal order judgement.

Importantly, Experiment 2 rules out explanations based on discomfort or awkwardness. If unusual or uncomfortable postures were in themselves responsible for poor temporal judgement, the doubly reversed posture of arm and hand should produce particularly poor performance. In fact, performance in this condition was no worse than an unreversed condition. Indeed, the total absence of main effects of either Elbow or Wrist angle suggests that the internal kinematic configuration of the body is largely irrelevant to temporal order judgement, as long as the external spatial location of the stimuli is preserved. One might say that time-perception is blind to the kinematic null-space of the postural control system (Haggard et al. 1995).

The results of Experiment 2 provide strong evidence that location of body parts in egocentric external space is automatically used for tactile perception, and in particular for temporal order judgement, even when irrelevant and detrimental. In order to retrieve and compare timings of two touches on the body, the brain used not only tactile afferent information, i.e. where the touch was located on the skin, but also information about the stimulus location in external space. Generating information about stimulus location in external space requires two integrative steps (Fig. 3). The process begins with a representation of the stimulus location on the receptor surface of the skin. This is first combined with information about the registration between skin locations and underlying body parts to generate a representation of stimulus location on the body. Second, somatotopic information about stimulus location on the body is

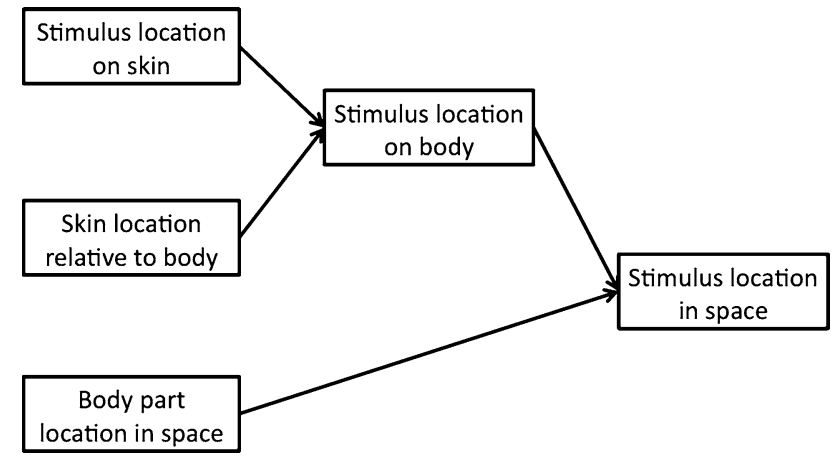

Fig. 3 Schematic model of tactile localisation process. The figure represents a schematic model of the possible steps involved in localising a tactile stimulus. See text for description

combined with proprioceptive information about the position of body parts in egocentric external space. This second process, often called tactile remapping, computes the position of the stimulus in egocentric external space. Our result suggests that temporal information is only available after this process has been completed.

\section{General discussion}

The ability to localise touches on the skin surface is essential for successful interaction with the surrounding environment. Here, we showed that the proprioceptive representation of body location in egocentric external space plays an important and automatic role in judging the position of touches on body surface.

Several previous studies using the temporal order judgement and spatial cueing paradigms showed that crossmodal links between vision and touch are updated with body part movement, to take current body posture into account (Kennett et al. 2001, 2002; Driver and Spence 1998; Eimer et al. 2001). These studies have generally investigated tactile remapping processes by crossing the hands across the body midline. This arrangement results in large and robust crossed hand effects. However, several features of the crossed hands arrangement may be rather specific and may not generalise to tactile remapping more widely. For example, some crossed hands deficits may include an element of left-right confusion at the response category stage. In addition, the crossed hands deficit might partly reflect a general difficulty in integrating information across the two cerebral hemispheres. Ours is the first study, to our knowledge, to manipulate body posture within a single hemispace and a single limb, and thereby show that representation of tactile stimuli in an external frame automatically dominates over purely somatotopic representations. Critically, the failure in the remapping process was not due to confusion regarding 
the identity of the two limbs, because only one limb was stimulated, and laterality was irrelevant. In addition, the response made reference to a body part defined anatomically, rather than spatially. Nevertheless, the current posture of the limb in egocentric space appeared to influence the perceived temporal order of touch.

Localisation of body parts in space therefore appears to be a fundamental step in tactile localisation process. Previous studies argued that tactile remapping process occurs automatically (Azanon and Soto-Faraco 2008a, b; Shore et al. 2005; Gallace and Spence 2005; Schicke and Röder 2006) even when it is maladaptive for the current task. Our results confirm that retrieval of external spatial locations of touch process is automatic. Our results extend knowledge of spatial remapping, by showing that proprioceptive information throughout the kinematic chain is accurately used to compute spatial location. That is, both elbow and wrist postures were considered for proprioceptive remapping of touch in Experiment 2. The forward kinematics of the entire limb chain appears to be computed before temporal information is accessed.

We found no interaction between Arm Posture and ISI in Experiment 1 and no interaction between ISI and either joint posture in Experiment 2. Although null results must be treated with caution in such small experiments, this finding seems worthy of discussion. The lack of an interaction suggests that limb posture and temporal event tagging act at different stages of a tactile localisation processing pathway. If posture had simply made tactile temporal order judgement more difficult, or impaired tactile temporal resolution, one might have expected stronger postural effects at shorter ISIs. In fact, the larger $550 \mathrm{~ms}$ ISI used in our study is well above the threshold level for temporal order judgement errors in other studies (Yamamoto and Kitazawa 2001a, b). Our finding of significant errors even for this easy temporal order judgement task may reflect the relative difficulty of temporal order judgement within a single body part, as compared to the conventional crossed hands arrangement. Moreover, in a recent study, de Haan et al. (2012) investigated how processing of tactile stimuli applied to the fingers would be affected by an unusual posture of the fingers (crossed fingers). They found that crossed finger positions did not affect performance, even at large SOAs up to $700 \mathrm{~ms}$. This seems to suggest that the localisation of stimuli in a somatotopic reference and the integration of this representation with postural information may be of greater importance for hands than for fingers, perhaps reflecting the differential mobility of these body parts.

More importantly, postural manipulations had similar effects on easy and on hard temporal judgements in both experiments of our study. Yamamoto and Kitazawa (2001a) have suggested that the external spatial location of touch is automatically accessed before temporal information can be obtained. Moreover, they also showed that conscious experience of touch is not necessarily bound to the location of mechanoreceptors on the skin that detect the touch. Particularly, they suggested that conscious sensation of touch is first to localised in external space where the hand is and then to the skin (Kitazawa 2002). Our results seem to confirm this view.

This form of sequential arrangement of space processing, followed by time processing, is consistent with the lack of interaction in our data. In Experiment 2, we found a modest trend for spatial postural effects to be weaker at higher ISIs, although we suggest that this may arise because of ceiling effects. Further studies of greater statistical power would be required to verify whether spatial and temporal effects are truly additive or not.

In summary, results from the present study confirmed that the localisation of a tactile stimulus requires first localising body parts in space. Several previous findings show that an external frame of reference is dominant in tactile spatial tasks, over and above the somatotopically based representations (Driver and Grossenbacher 1996; Vallar 1997; Pavani et al. 2000; Eimer et al. 2001; Maravita et al. 2002a, b; Spence et al. 2003; Zampini et al. 2005). Interestingly, spatial localisation of touch is necessary even in a task that makes no explicit reference to external spatial location, but only to body landmarks. The model of somatoperceptual information processing of Longo et al. (2010) provides a hierarchical model for tactile localisation. Particularly, it first requires a somatic localisation of touch on a skin map. This process is entirely independent of body posture. Second, skin regions are registered with the body parts that lie beneath them. This process is largely independent of body posture as well. Skin stretch and tissue displacement during movement can change the spatial relation between skin and musculoskeletal structures, but not enough to explain the errors produced by our participants. Changes in posture have been suggested to change the arrival time of stimuli at the brain (see Bergenheim et al. 1996). Body part posture certainly affects some aspects somatosensory processing (Soto-Faraco et al. 2004; Overvliet et al. 2011), particularly when the posture is unusual. However, a strong point of our study is that participants were never asked to compare the time of occurrence of tactile stimuli presented across different body parts, but only tactile stimuli presented on a single body part, on one side of the body. Third, body part locations are represented in an external frame of reference on the basis of proprioceptive inputs, which are updated as the limbs move through space. This third step has been called tactile remapping. Our results show that tactile remapping occurs automatically even when maladaptive and irrelevant to the task. Our results also confirm that tactile remapping operates within a single hemisphere and follows the proprioceptively signalled kinematics of the entire limb. 
Conscious perception of time seems fundamentally linked to location of stimuli in egocentric external space and cannot access the brain representations housing pure somatotopic sensation, even when required to do so.

Acknowledgments E.R.F. and P.H. were supported by EU Project VERE Work Package 1. P.H. was additionally supported by ERC Advanced Grant HUMVOL.

Conflict of interest The authors declare that the research was conducted in the absence of any commercial or financial relationships that could be construed as a potential conflict of interest.

\section{References}

Azanon E, Soto-Faraco S (2008a) Changing reference frames during the encoding of tactile events. Curr Biol 18(14):1044-1049

Azanon E, Soto-Faraco S (2008b) Spatial remapping of tactile events: assessing the effects of frequent posture changes. Commun Integr Biol 1(1):45-46

Azanon E, Camacho K, Soto-Faraco S (2010a) Tactile remapping beyond space. Eur J Neurosci 31(10):1858-1867

Azanon E, Longo MR, Soto-Faraco S, Haggard P (2010b) The posterior parietal cortex remaps touch into external space. Curr Biol 20(14):1304-1309

Bergenheim M, Johansson H, Granlund B, Pedersen J (1996) Experimental evidence for a sensory synchronization of sensory information to conscious experience. In: Hameroff SR, Kaszniak AW, Scott AC (eds) Toward a science of consciousness: the first Tuscon discussions and debates. MIT Press, Cambridge, MA, pp 303-310

Bisiach E, Perani D, Vallar G, Berti A (1986) Unilateral neglect: personal and extra-personal. Neuropsychologia 24(6):759-767

de Haan AM, Anema HA, Dijkerman HC (2012) Fingers crossed! An investigation of somatotopic representations using spatial directional judgements. PLoS One 7(9):e45408

Driver J, Grossenbacher PG (1996) Multimodal spatial constraints on tactile selective attention. In: Inui T, McClelland JL (eds) Attention and performance, XVI: information integration in perception and communication. MIT Press, Cambridge, MA, pp 209-235

Driver J, Spence C (1998) Cross-modal links in spatial attention. Philos Trans R Soc Lond B Biol Sci 353(1373):1319-1331

Eimer M, Cockburn D, Smedley B, Driver J (2001) Cross-modal links in endogenous spatial attention are mediated by common external locations: evidence from event-related brain potentials. Exp Brain Res 139(4):398-411

Flach R, Haggard P (2006) The cutaneous rabbit revisited. J Exp Psychol Hum Percept Perform 32(3):717-732

Gallace A, Spence C (2005) Visual capture of apparent limb position influences tactile temporal order judgments. Neurosci Lett 379(1):63-68

Haggard P, Hutchinson K, Stein J (1995) Patterns of coordinated multi-joint movement. Exp Brain Res 107(2):254-266

Head H, Holmes G (1911) Sensory disturbances from cerebral lesions. Brain 34(2-3):102
Husain M (2008) Hemispatial neglect. In: Goldenberg G, Miller BL (eds) Handbook of clinical neurology. Elsevier, Amsterdam, pp 359-372

Kennett S, Eimer M, Spence C, Driver J (2001) Tactile-visual links in exogenous spatial attention under different postures: convergent evidence from psychophysics and ERPs. J Cogn Neurosci 13(4): $462-478$

Kennett S, Spence C, Driver J (2002) Visuo-tactile links in covert exogenous spatial attention remap across changes in unseen hand posture. Percept Psychophys 64(7):1083-1094

Kitazawa S (2002) Where conscious sensation takes place. Conscious Cogn 11:475-477

Longo MR, Azanon E, Haggard P (2010) More than skin deep: body representation beyond primary somatosensory cortex. Neuropsychologia 48(3):655-668

Maravita A, Clarke K, Husain M, Driver J (2002a) Active tool use with the contralesional hand can reduce cross-modal extinction of touch on that hand. Neurocase 8(6):411-416

Maravita A, Spence C, Kennett S, Driver J (2002b) Tool-use changes multimodal spatial interactions between vision and touch in normal humans. Cognition 83(2):B25-B34

Overvliet KE, Anema HA, Brenner E, Dijkerman HC, Smeets JB (2011) Relative finger position influences whether you can localize tactile stimuli. Exp Brain Res 208(2):245-255

Pavani F, Spence C, Driver J (2000) Visual capture of touch: outof-the-body experiences with rubber gloves. Psychol Sci 11(5):353-359

Penfield W, Rasmussen T (1950) The cerebral cortex of man. A clinical study of localization of function. MacMillan, New York

Röder B, Rösler F, Spence C (2004) Early vision impairs tactile perception in the blind. Curr Biol 14(2):121-124

Schicke T, Röder B (2006) Spatial remapping of touch: confusion of perceived stimulus order across hand and foot. Proc Natl Acad Sci U S A 103(31):11808-11813

Shore DI, Gray K, Spry E, Spence C (2005) Spatial modulation of tactile temporal-order judgments. Perception 34(10):1251-1262

Soto-Faraco S, Ronald A, Spence C (2004) Tactile selective attention and body posture: assessing the multisensory contributions of vision and proprioception. Percept Psychophys 66(7):1077-1094

Spence C, Baddeley R, Zampini M, James R, Shore DI (2003) Multisensory temporal order judgements: when two locations are better than one. Percept Psychophys 65(2):318-328

Vallar G (1997) Spatial frames of reference and somatosensory processing: a neuropsychological perspective. Philos Trans R Soc Lond B Biol Sci 352(1360):1401-1409

Weinstein S (1968) Intensive and extensive aspects of tactile sensitivity as a function of body part, sex and laterality. In: Kenshalo DR (ed) The skin senses. Thomas, Springfield, IL, pp 195-222

Yamamoto S, Kitazawa S (2001a) Reversal of subjective temporal order due to arm crossing. Nat Neurosci 4(7):759-765

Yamamoto S, Kitazawa S (2001b) Sensation at the tips of invisible tools. Nat Neurosci 4(10):979-980

Zampini M, Harris C, Spence C (2005) Effect of posture change on tactile perception: impaired direction discrimination performance with interleaved fingers. Exp Brain Res 166(3-4):498-508 